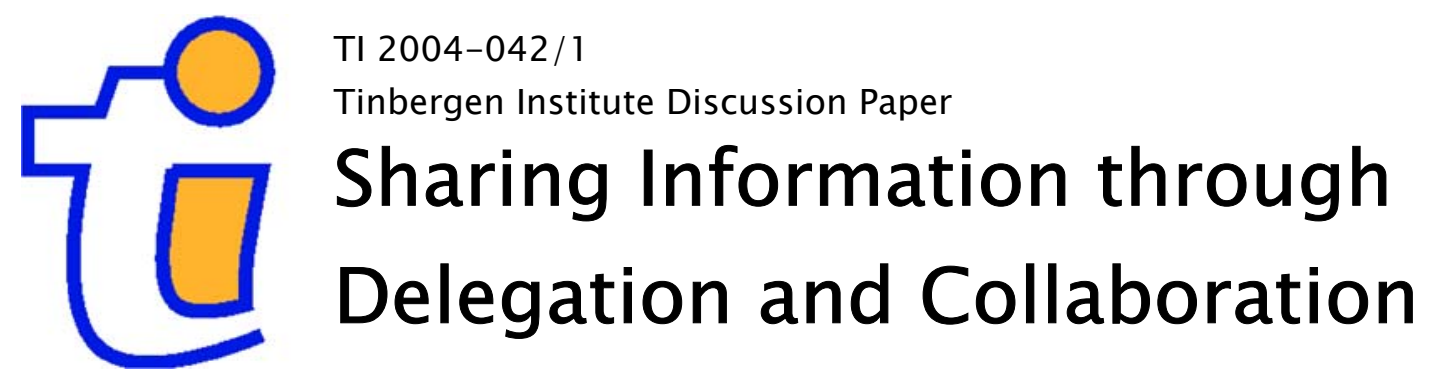

Otto H. Swank

Bauke Visser

Department of Economics, Erasmus Universiteit Rotterdam, and Tinbergen Institute. 


\section{Tinbergen Institute}

The Tinbergen Institute is the institute for economic research of the Erasmus Universiteit Rotterdam, Universiteit van Amsterdam, and Vrije Universiteit Amsterdam.

Tinbergen Institute Amsterdam

Roetersstraat 31

1018 WB Amsterdam

The Netherlands

Tel.: $\quad+31(0) 205513500$

Fax: $\quad+31(0) 205513555$

Tinbergen Institute Rotterdam

Burg. Oudlaan 50

3062 PA Rotterdam

The Netherlands

Tel.: $\quad+31(0) 104088900$

Fax: $\quad+31(0) 104089031$

Please send questions and/or remarks of nonscientific nature to driessen@tinbergen.nl.

Most TI discussion papers can be downloaded at http://www.tinbergen.nl. 


\title{
Sharing Information through Delegation and
}

\section{Collaboration*}

\author{
Otto H. Swank \\ Erasmus University Rotterdam \\ and Tinbergen Institute
}

\author{
Bauke Visser ${ }^{\dagger}$ \\ Erasmus University Rotterdam \\ and Tinbergen Institute
}

April 8, 2004

\begin{abstract}
This article analyzes under which conditions a manager can motivate a junior worker by verbal communication, and explains why communication is often tied up with organizational choices as job enlargement and collaboration. Our model has two important features. First, the manager has more information about a junior's ability than the junior himself. Second, the junior's effort and ability are complements. We show that the manager has an incentive to exaggerate the junior's ability. We discuss two ways in which the manager can make credible statements about the junior's ability. First, the senior can delegate a task to the
\end{abstract}

*We are grateful to Marijke Bellaar for providing information about the way medical specialists supervise their students at the Faculty of Medicine at Utrecht University; to Bas Heijs for informing us about the content of management courses offered by the Dutch Ministry of Finance; to Job Swank for information on the role played by heads of departments and managers at the Dutch Central Bank; and to Jose Luis Moraga Gonzalez for helping us with the graphs.

${ }^{\dagger}$ Corresponding author. Erasmus University Rotterdam, H 7 - 20, P.O. Box 1738, 3000 DR Rotterdam, The Netherlands. Email: bvisser@few.eur.nl 
junior for which it is important that the junior has a correct perception of his ability. Information is shared through a costless signal. Second, the senior can spend more time on a junior she perceives as able than on a junior she perceives as less able. Information is then shared through a costly signal.

Keywords: Communication, incentives, signalling, overconfidence, delegation, collaboration

JEL codes: C70, D23, D83

\section{Introduction}

Motivating colleagues is an important role of superiors. When analyzing this role, economists tend to focus on monetary incentives and rewards. ${ }^{1}$ Consequently, the multi-faceted relationship between superior and subordinate is often reduced to a contractual one. In particular, verbal communication is by and large ignored although it is likely to take place on a daily basis and aims at exerting influence on a subordinate's attitude and at inspiring desirable behaviour. In this paper, we analyse under what conditions verbal communication motivates, and explain why communication is often connected with organizational choices, such as job enlargement and collaboration.

A few examples illustrate what we intend to capture. In 2003, the governing board of the Dutch Central Bank explicitly formulated its view on the role of managers in a document entitled 'Management Competencies'. ${ }^{2}$ In this view, managers are supposed to motivate subordinates by acting as a mentor. Managers should challenge subordinates by delegating tasks, taking into account the risk for the Bank. Moreover, managers should be able to assess the abilities of their subordinates and assign tasks and responsibilities accordingly. ${ }^{3}$

\footnotetext{
${ }^{1}$ For a survey see, e.g., Prendergast (1999).

2 'Managementcompetenties' in Dutch.

${ }^{3}$ Monetary rewards are also used to motivate at the Dutch Central Bank. The extent to which a
} 
Managers at the Dutch Ministry of Finance are required to follow management courses. In these courses, the manager is viewed as a coach. These courses pay substantial attention to subjects like attention, perception and delegation. Finally, a cursory examination of various MBA programmes suggests that in these programmes discussions of communication, persuasion, and leadership are at least as common as analyses of compensation schemes. ${ }^{4}$

To analyse the possibilities and limits of communication as a means of motivating, we present a model in which an agent ('he') designs a project that is then submitted to the principal ('she') who decides whether to implement it or not. Both the principal and the agent care about the success of the project. We assume that the principal has a better assessment of the agent's ability to design a successful project than the agent himself. One can think of the principal as an experienced manager or head of department who is responsible for ensuring that the agent contributes to the organization. Their relationship could be one of supervision, as when the agent is a newly recruited employee like a management trainee. But one could equally well think of an experienced (associate) professor and an assistant professor.

Although it is an essential feature of our model that the principal has better information than the agent, we do not want to claim that this is always the case. For example, when an experienced agent gets to a new job that is very similar to his previous one, he has probably a more accurate view of his ability than his new principal. However, when the agent is young, his self-assessment is based on limited professional experience, if any, while his principal may have much experience of assessing young agents. Thus, our model typically describes situations in which the agent is at the manager's daily interactions with subordinates provide testimony to the desired competencies has an influence on the manager's bonus (Job Swank, personal communication).

${ }^{4}$ We had a look at the courses offered at Wharton School of Business, Chicago Graduate School of Business, Harvard Business School, Rotterdam School of Management, and London Business School. 
beginning of his career or performs tasks for the first time, while the principal has had experience with a number of similar agents. For this reason we refer to the agent as the 'junior' and to the principal as the 'senior'.

Another important feature of our model is that a junior's effort depends positively on his belief that he is able: ability and effort are complements. In particular, the more able is a junior, the higher is the likelihood that his effort leads to a good project. An implication is that the senior, having an interest in a good result, has an incentive to make the junior believe he is able. That is, once the senior has formed an impression of the junior that is not all too favourable, she cannot escape exaggerating his ability. However, the junior sees through the senior's intentions and rationally discounts the senior's claim. In our model this discounting induces the junior to ignore statements made by the senior. Thus, communication alone does not motivate. It is merely cheap talk.

Although the senior cannot be withheld from making exaggerated claims so as to boost the agent's confidence in his ability, a priori she would have preferred to commit herself to truthfully revealing her assessment. The extra effort of the more able junior and the concomitant increased likelihood of a good project more than outweighs the reduction in effort stemming from the realisation that one is not as able as initially thought. That is, truthfully reporting what the senior knows about the junior's ability leads to the first best situation. By exaggerating in case of a relatively bad impression, she destroys the possibility of convincingly claiming a truly favourable assessment. In our model, then, stimulating self-confidence prevents the creation of self-knowledge.

We analyse two organizational choices that can be interpreted as ways of making communication credible. The first is job enlargement through delegation of authority. By this we mean that the junior is allowed to both design the project and to decide on its future. By claiming to delegate only if the senior's assessment is favourable, the 
temptation is to delegate also in case of a less favourable impression of the junior's ability as this would stimulate effort. We refer to this increase in effort as the motivation effect. However, exaggerating the junior's ability also causes him to become too confident when it comes to project implementation. Thinking he is more able than he really is, and therefore that a project is more likely to be successful than it actually will be, a project is implemented under conditions the senior would have discarded it. This negative effect could be called the 'overconfidence effect' of exaggeration. If this effect dominates the motivation effect, the senior is not tempted to exaggerate the perceived ability of the junior. In this situation, delegation enables credible communication. Moreover, it does so at no costs. The first best can be attained because of the presence of costs that are latent and that only become manifest in case of delegating to a less able junior.

Our model of delegation describes how senior medical specialists supervise their students. In many countries, training as a medical specialist is received by means of hospital internships under the supervision of senior specialists. Seniors are generally much better able to assess their students' skills than the students themselves. The students infer information about their abilities from the way the senior delegates tasks. A student who is only allowed to perform simple operations will conclude that he is less able than a student who is allowed to perform more complicated operations. When deciding whether or not to delegate a specific treatment to a student, the senior is aware that her decision affects the student's perception of his abilities.

A second way in which communication can be made credible is by having the senior selectively dedicate time and attention to the design of the project or to the junior himself. The senior may be able to truthfully reveal her information, by engaging herself in the design phase only if she has a favourable impression of the junior's ability to develop a successful project. As Mintzberg (1971) found in his empirical 
analysis of managerial work, this engagement can take on various forms. The senior may forward to the junior useful information she obtains from peers or other parts of the organization; she may ensure the junior can work in an especially pleasant environment and provide encouragement; she can inform interested parties of the project that is under development and thereby create a more productive environment in which to design the project; and obviously she can spend time and effort on the design of the project herself - e.g. preparatory, supervisory or collaborative effort. ${ }^{5}$

We modify our base model by introducing the possibility that the senior's effort affects the likelihood of success of the project. The junior's effort is still a prerequisite for possible project success. From the productive point of view, i.e. barring communication problems, she would help more if she has a favourable impression of the junior than a bad one, because in the former case her effort is more likely to be productive than in the latter. The temptation for the senior is once again to make a junior of whom she has an unfavourable impression believe she in fact thinks he is very able by being as collaborative as in case of a positive assessment. What may prevent the senior from giving in to this temptation are the costs of time and effort that would be exceedingly high in comparison with the increase in the likelihood of successful project design. The larger the difference between the effort levels from a productive point of view in case of a favourable and an unfavourable assessment, the more likely it is that this temptation does not exist. However, if this difference is too small, credible communication requires more effort in case the junior is truly thought to be able. This distorts the effort decisions in case of a favourable assessment. The senior becomes too engaged, e.g. by overly pampering the junior or by putting him under excessive stress. As a result, the latter also dedicates too much time to project development. Similarly,

\footnotetext{
${ }^{5}$ The various forms of engagement are described by Mintzberg (1971) as roles. Here we refer to the role of disseminator of information and as a liaison figure, leadership, spokesman, and resource allocator, respectively.
} 
if the senior's effort does not directly influence the likelihood of success of the project but amounts to providing attention to the junior, its discriminatory use can work as a credible signal.

Our paper contributes to the voluminous literature on organizational design. This literature deals with a wide variety of issues, such as the debate about centralization versus decentralization, the allocation of tasks and responsibilities, and problems concerning communicating and processing information, to mention a few. The literature focusing on the relationship between the assignment of tasks and communication emphasizes the way in which task assignment influences the nature and amount of communication between departments to attain a desirable organizational outcome (e.g. Marschak and Radner 1972) and stresses the desirability of hierarchical structures from a communication and information processing point of view (e.g. Calvo and Wellisz 1978, Garicano 2000). We differ from either line of research. A distinguishing feature of our approach is that the assignment of tasks makes communication possible in the first place rather than that it influences the total cost of communication or the speed with which a final decision is reached. Of course, we are aware that the allocation of tasks also has important implications for collecting and processing information.

More specifically, our paper is related to studies describing situations in which a principal has more information about a key aspect of the production process than an agent. One of those studies is Benabou and Tirole (2002), which, like ours, analyzes a model in which a senior has better information about the junior's ability than the junior himself, and effort and ability are complements. Concerning delegation and collaboration, the focus of their analysis is on how the senior's decision whether or not to delegate a task (or whether or not to collaborate) affects a worker's perception of himself, and in turn influences his effort. Unlike us, Benabou and Tirole assume that a sorting condition holds. This sorting condition guarantees that the junior can infer 
information about his ability from the senior's decision to delegate or to collaborate. Our focus is on the conditions under which delegation and collaboration can be used as a means of communicating information about the junior's ability. We show that through her decision about the allocation of tasks, a senior can sometimes reveal information. We thus offer a new rationale for delegation and collaboration. ${ }^{6}$ Our model of collaboration is in spirit most similar to Hermalin's (1998) model of leadership. In that model, one worker in a team, the leader, has better information about the benefits of a common project. The leader cannot share the information with the other workers through ordinary talk. Hermalin shows how a leader can signal her information by exerting costly effort. Like us, he focuses on the characteristics of the equilibrium in which credible communication takes place. An advantage of our approach over Hermalin's and Benabou and Tirole's is that it yields predictions about the circumstances under which a senior chooses delegation or collaboration as a means of revealing her information about the junior's ability.

We find that communication through delegation requires that the senior has a fairly accurate view of the junior's ability. The reason is that when a senior is quite certain that the junior is not able, overconfidence constitutes a major risk. Consequently, a senior who accurately assesses a junior's ability has hardly an incentive to delegate the decision about the project to a junior she assesses not able. In case the senior does not have an accurate view of the junior's ability, delegation does not work as a means of communication. The benefits of higher effort levels exceed the costs arising from overconfidence. In that case, attention (or collaboration) as a means of communication usually remains possible.

The rest of the paper is organized as follows. In section 2 we present the base com-

\footnotetext{
${ }^{6}$ There are other reasons for delegation, such as a better utilization of information at low levels of hierarchies. Delegation may also induce agents to exert more effort (see e.g. Aghion and Tirole, 1997; Zábojnik, 2002).
} 
munication model which we analyse in section 3. In section 4 we discuss the conditions under which delegation makes credible communication possible. Then, in section 5 , we analyse the circumstances under which collaboration overcomes the senior's credibility problem. Section 6 discusses the role attention can play. Section 7 concludes. Proofs can be found in the appendix.

\section{The Base Communication Model}

Consider a situation where two tasks have to be performed. The first task is the design of a project. The second task is to decide whether to implement the project, $X=1$, or not, $X=0$. The task of designing a project is delegated to a risk-neutral individual to which we refer as the junior ('he'). A designed project is either of good or bad quality. The probability that a junior designs a good project depends on his ability, $a$. The junior can be smart or dumb, $a \in\{S M, D U\}$. The prior probability with which the junior thinks he is smart equals $\eta$. A smart junior differs from a dumb one in that the effort the former puts in the design of a project increases the probability that the project is good, while a dumb agent always designs a bad project, irrespective of the effort he exerts. The probability that a smart agent designs a good project is denoted by $\pi(e)$, where $e$ denotes effort. We assume that $\pi(0)=0, \pi^{\prime}>0$ and $\pi^{\prime \prime} \leq 0$. The junior is effort averse. The costs of effort are represented by $c(e)$, with $c(0)=0, c^{\prime}>0$, and $c^{\prime \prime}>0$.

Once the project has been designed, a decision has to be taken on the project. In the base model, we assume that the junior's superior (senior, 'she') takes this decision. Whether or not the project should be implemented depends on the quality of the project and on the state of the world. If the project is good, then the project yields a payoff to the junior and his senior equal to $p+f+\mu$, with $f>0$. If the project is bad, it yields a payoff equal to $p+\mu$. After a project has been designed, Nature draws 
$\mu$ from a uniform distribution with support $[-h, h]$. Both the junior and his senior observe $\mu$. A good (bad) project should be implemented if $p+f+\mu>0(p+\mu>0)$. We assume that $p+f-h<0$ and $p+h>0$. The implication of these assumptions is that the decision about the project should depend on the realization of $\mu .^{7}$

The senior receives a noisy signal $s=\{s m, d u\}$ about the junior's ability. With probability $\beta$, the signal is fully informative, that is $\operatorname{Pr}(a=S M \mid s=s m$, info $)=1$ and $\operatorname{Pr}(a=D U \mid s=d u$, info $)=1$. With probability $1-\beta$, the signal is uninformative: $\operatorname{Pr}(a=S M \mid s=s m$, uninfo $)=\eta$ and $\operatorname{Pr}(a=D U \mid s=d u$, uninfo $)=1-\eta$. The senior does not know whether the signal is informative or not, but does know the value of $\beta$. Thus, $\gamma_{s m}=\operatorname{Pr}(a=S M \mid s=s m)=\beta+(1-\beta) \eta$ and $\gamma_{d u}=\operatorname{Pr}(a=S M \mid s=d u)=$ $(1-\beta) \eta$. For $\beta>0$ we have that $\gamma_{s m}>\eta>\gamma_{d u}$. As the junior does not know the signal $s$ his senior received, the senior can be of two types $s$. The junior does know the accuracy $\beta$ of the signal. After the senior has received her signal, but before the junior designs a project, the senior sends a message $m$ to the junior. The message is either $m=s m$, meaning 'I have received a signal $s=s m$ ', or $m=d u$, meaning 'I have received a signal $s=d u^{\prime}$.

\footnotetext{
${ }^{7}$ Our assumption that, apart from costs, the payoff of the junior and the senior are identical is not material for the nature of our results. It would be sufficient for the payoffs to be positively correlated. What is essential is that both junior and senior are motivated by the fruits of their work - either because of an intrinsic interest in their accomplishments or as a way of improving their (individual) lot in the future.
} 


\section{Table 1 The Base Communication Game}

Players: junior and senior

\section{Sequence of the game}

- Nature chooses the ability of junior, $a \in\{D U, S M\}$. With probability $\eta, a=S M$ and with probability $(1-\eta), a=D U$.

- The senior receives a signal $s \in\{d u, s m\}$ about $a: \operatorname{Pr}(a=S M \mid s=s m)=\gamma_{s m}$ $>\eta$ and $\operatorname{Pr}(a=S M \mid s=d u)=\gamma_{d u}<\eta$.

- The senior sends a message about her signal to the junior: $m \in\{d u, s m\}$.

- junior chooses effort $e \geq 0$ at cost $c(e)$ and designs a project.

- If $a=S M$, then the expected benefit of the project equals $p+f$ with probability $\pi(e)$ and $p$ with probability $1-\pi(e)$. If $a=D U$, then the expected benefit of the project equals $p$.

- Nature draws $\mu$ from a uniform distribution function with $\mu \in[-h, h] . \mu$ is revealed to both the junior and the senior.

- The senior takes a decision on the project, $X \in\{0,1\}$.

\section{Payoffs:}

- junior: $U(X=0)=-c(e) ; U(X=1 \mid$ Good project $)=p+f+\mu-c(e)$; $U(X=1 \mid$ bad project $)=p+\mu-c(e)$.

- Superior: $U(X=0)=0 ; U(X=1 \mid$ Good project $)=p+f+\mu ; U(X=1 \mid$ bad project $)=p+\mu$. 
In the next section we characterize the two-period perfect Bayesian equilibrium. This amounts to a strategy for the junior that is optimal given his belief about the type of senior; a strategy for either type of senior that is optimal given the strategy of the junior and the strategy of the other type of senior; and a belief held by the junior about the type of senior that is consistent with the senior's type-dependent strategies. In our game, the senior's message is a costless, nonverifiable claim; it is cheap talk. It is well-known that in cheap-talk games, a babbling equilibrium always exists. In such an equilibrium, the sender of the message randomly chooses a message and the receiver ignores the message. Clearly, these strategies are optimal responses to each other. In this paper, we ignore the babbling equilibrium.

\section{Analyzing the Base Model}

In this section, we make the point that the senior cannot avoid exaggerating the perceived ability of the junior, i.e., she cannot avoid sending $m=s m$, 'I have received a signal $s=s m$, if in reality $s=d u$. The intuition is the following. Suppose the junior believes the senior truthfully reveals her information. Then, by sending a message $m=s m$ rather than $m=d u$, the senior enhances the junior's confidence in his ability,

which in turn increases the marginal revenue of his effort. We call this the 'motivation effect' of the message. The junior understands this temptation and therefore ignores the message $m=s m$ and instead bases his effort decision on his prior belief $\eta$ about his ability.

To solve our game, first consider the implementation decision. Conditional on the senior having received signal $s$, implementation of the project yields a higher payoff than maintaining status quo if

$$
\gamma_{s} \pi(e)(p+f+\mu)+\left[1-\gamma_{s} \pi(e)\right](p+\mu)>0
$$


Clearly, the senior implements the project if $\mu>\mu_{s}(e)$, where

$$
\mu_{s}(e)=-p-\gamma_{s} \pi(e) f
$$

To ensure that the implementation decision is important we assume:

Assumption $1\left|\mu_{s}(e)\right|<h$ for $s \in\{d u, s m\}$

Let us now consider the problem the junior faces. The junior determines how much effort to put in designing a project as a function of the message $m$ the senior sends. In making this decision, the junior anticipates that the senior will make the implementation decision on the basis of Eq (2). Suppose the junior believes that the senior truthfully reveals her information, i.e., a senior of type $s$ sends message $m=s$. Consistent with this strategy of both types of senior is the junior's belief that $s=m$, so that

$$
\begin{aligned}
e_{m}^{*} & =\arg \max _{e} \operatorname{Pr}\left(\mu>\mu_{m}(e)\right) E\left(p+\gamma_{m} \pi(e) f+\mu \mid \mu>\mu_{m}\left(e_{s}\right)\right)-c(e) \\
& =\arg \max _{e} \operatorname{Pr}\left(\mu>\mu_{m}(e)\right)\left(-\mu_{m}(e)+\frac{1}{2}\left(h+\mu_{m}(e)\right)\right)-c(e) \\
& =\arg \max _{e} \frac{1}{4 h}\left(p+h+\gamma_{m} \pi(e) f\right)^{2}-c(e)
\end{aligned}
$$

Consequently, in case of an interior solution, $e_{m}^{*}$ satisfies the first-order condition ${ }^{8}$

$$
\frac{1}{2 h} \pi^{\prime}(e) \gamma_{m} f\left(p+h+\gamma_{m} \pi(e) f\right)=c^{\prime}(e)
$$

We assume that in case $s=s m$ such an interior solution exists:

Assumption 2 If the junior believes that the senior truthfully reveals her information, then, in case of $s=s m, \frac{1}{2 h} \pi^{\prime}(0) \gamma_{s m} f\left(p+h+\gamma_{s m} \pi(0) f\right)>c^{\prime}(0)$ such that $e_{s m}^{*}>0$.

\footnotetext{
${ }^{8}$ The second-order condition is: $\frac{1}{2 h} \pi^{\prime \prime}(e) \gamma_{m} f\left(p+h+\gamma_{m} \pi(e) f\right)+\frac{1}{2 h}\left(\pi^{\prime}(e)\right)^{2} \gamma_{m}^{2} f^{2}-c^{\prime \prime}(e)<0$.
} Throughout, it is assumed that this condition is satisfied. 
The following lemma results from application of the implicit function theorem to Eq (4).

Lemma 1 Suppose the junior believes the senior, i.e., $m=s$. Then $e_{s m}^{*}>e_{d u}^{*}$. Moreover, for given $m$, the junior's effort is increasing in $p, f, \gamma_{d u}, \gamma_{s m}$ and a decreasing function of $h$.

The intuition behind the results presented in Lemma 1 is as follows. If the junior believes the senior's announcement, then the message $m=s m$ rather than $m=d u$ increases the likelihood that the junior is smart and that therefore his effort will be likely productive. As a result, the junior exerts more effort. Effort increases with $f, p, \gamma_{s m}$ and $\gamma_{d u}$ as $f$ is the potential benefit of effort, and a rise in $p, \gamma_{s m}$ or $\gamma_{d u}$ increases the probability that the fruits of effort can be reaped. A rise in $h$ implies that the realization of $\mu$ becomes more important for the implementation decision. The implication is that the gain of effort relatively decreases. An increase in $h$ thus decreases effort.

Still assuming that the junior believes that the senior truthfully reveals her information, the senior's payoff if she receives signal $s$ and sends message $m=s$ becomes

$$
\frac{1}{4 h}\left(h+p+\gamma_{s} \pi\left(e_{m}^{*}\right) f\right)^{2}
$$

It immediately follows that the senior has an incentive to exaggerate the ability of the junior (i.e, tell $m=s m$ in case $s=d u$ ). Of course, a direct implication is that the junior will ignore the senior's message. The message does not contain any information about the junior's ability.

Lemma 2 In the base communication game, a separating equilibrium in which the senior truthfully reveals her information does not exist.

In the remainder of this paper we will focus on situations in which the senior's incentive to exaggerate the junior's ability is a problem in the sense that ex ante, the 
senior would benefit from always telling the truth. The reason that the senior wants to share her information is that the junior would exert more effort in case it were most likely to be productive $(s=s m)$. This raises the question whether the organization of work can be adapted so that the senior can credibly convey information about the junior's ability. In the next two sections we analyse two ways in which credible communication can take place. In Section 4, we analyse how delegating the decision to implement the project to the junior may allow the senior to signal her information. In Section 5, we turn to the possibility of collaboration between the senior and the junior in the project design stage as a means of communication.

\section{Delegation of the Implementation Decision}

In this section, we extend the base model by giving the senior the opportunity to delegate the implementation decision to the junior. Formally, the senior can choose $D=1(D=0)$, meaning that the junior (senior) will make the decision on the project. The senior's delegation decision is observed by the junior and takes place after the senior received signal $s$, but before the junior chooses effort. We assume that the senior cannot renege on her delegation decision. That is, if she chooses $D=1$, it is the junior who in fact makes the implementation decision. We focus on the case in which delegation of the implementation decision $(D=1)$ means "junior, my assessment is you are smart with probability $\gamma_{s m}$ ", and not delegating $(D=0)$ means "junior, my assessment is you are smart with probability $\gamma_{d u}$ ". With little loss of generality we assume that the delegation decision automatically implies $m=s m(m=d u)$. In other words, the senior communicates by delegating. ${ }^{9}$

The following proposition gives the condition under which delegation of the imple-

\footnotetext{
${ }^{9}$ The only implication is that we do not have to specify out-of-equilibrium beliefs when $(D=1, m=d u)$ or $(D=0, m=s m)$.
} 
mentation decision solves the senior's credibility problem.

\section{Proposition 1 If}

$$
\begin{aligned}
& \frac{1}{4 h}\left(p+h+\pi\left(e_{d u}^{*}\right) \gamma_{d u} f\right)^{2}> \\
& \frac{1}{2 h}\left(p+h+\pi\left(e_{s m}^{*}\right) \gamma_{s m} f\right)\left(p+\pi\left(e_{s m}^{*}\right) \gamma_{d u} f+\frac{1}{2}\left(h-p-\pi\left(e_{s m}^{*}\right) \gamma_{s m} f\right)\right)
\end{aligned}
$$

then a separating equilibrium exists, in which the senior chooses $D=1$ if $s=s m$, and $D=0$ if $s=d u$; The project is implemented if and only if

$$
\mu>-p-\pi\left(e_{s}^{*}\right) \gamma_{s} \text { fwith } s \in\{d u, s m\}
$$

where $e_{s}^{*}$ solves

$$
\frac{1}{2 h} \pi^{\prime}\left(e_{s}\right) \gamma_{s} f\left[p+h+\pi\left(e_{s}\right) \gamma_{s} f\right]=c^{\prime}\left(e_{s}\right)
$$

The junior's beliefs are $\operatorname{Pr}(s=s m \mid D=1)=1$ and $\operatorname{Pr}(s=d u \mid D=0)=1$.

Eq (6) gives the condition under which the senior has no incentive to delegate the implementaton decision to the junior when $s=d u$. The left hand side gives the senior's payoff if she does not delegate the implementation decision to the junior. The right hand side gives the payoffs that would result from delegating the implementation decision to the junior. The costs and benefits of delegation to a junior in case of $s=d u$ can be seen from the right hand side. Delegation would lead the junior to hold an exaggerated belief in his ability. This gives rise to an increase in effort from $e_{d u}^{*}$ to $e_{s m}^{*}$. This captures the motivation effect of exaggerating ability. However, the implementation decision would now also be based on an inflated belief in the junior's ability. The junior would be too confident that the project will be successful and would therefore implement the project in situations in which the senior would reject the project. This overconfidence effect can be seen from the use of $\gamma_{s m}$ rather than $\gamma_{d u}$ in the probability of implementation, $\operatorname{Pr}\left(\mu>-p-\pi\left(e_{s m}^{*}\right) \gamma_{s m} f\right)=\frac{p+h+\pi\left(e_{s m}^{*}\right) \gamma_{s m} f}{2 h}$, and in the expected value of $\mu$ of a project that is implemented, $\frac{1}{2}\left(h-p-\pi\left(e_{s m}^{*}\right) \gamma_{s m} f\right)$. 
It follows from Eq (6) that if the senior's signal is highly accurate, delegation of the implementation decision works as a credible communication device. For $\beta=1$, $\gamma_{s m}=\beta+(1-\beta) \eta=1$ and $\gamma_{d u}=(1-\beta) \eta=0$. Then, Eq (6) amounts to

$$
\frac{1}{4 h}(p+h)^{2}>\frac{1}{4 h}\left(p+h+\pi\left(e_{s m}^{*}\right) f\right)\left(p+h-\pi\left(e_{s m}^{*}\right) f\right)
$$

which generally holds true. By continuity of $e_{s m}^{*}$ and $e_{d u}^{*}$ in $\beta$ this inequality also holds for sufficiently large values of $\beta$.

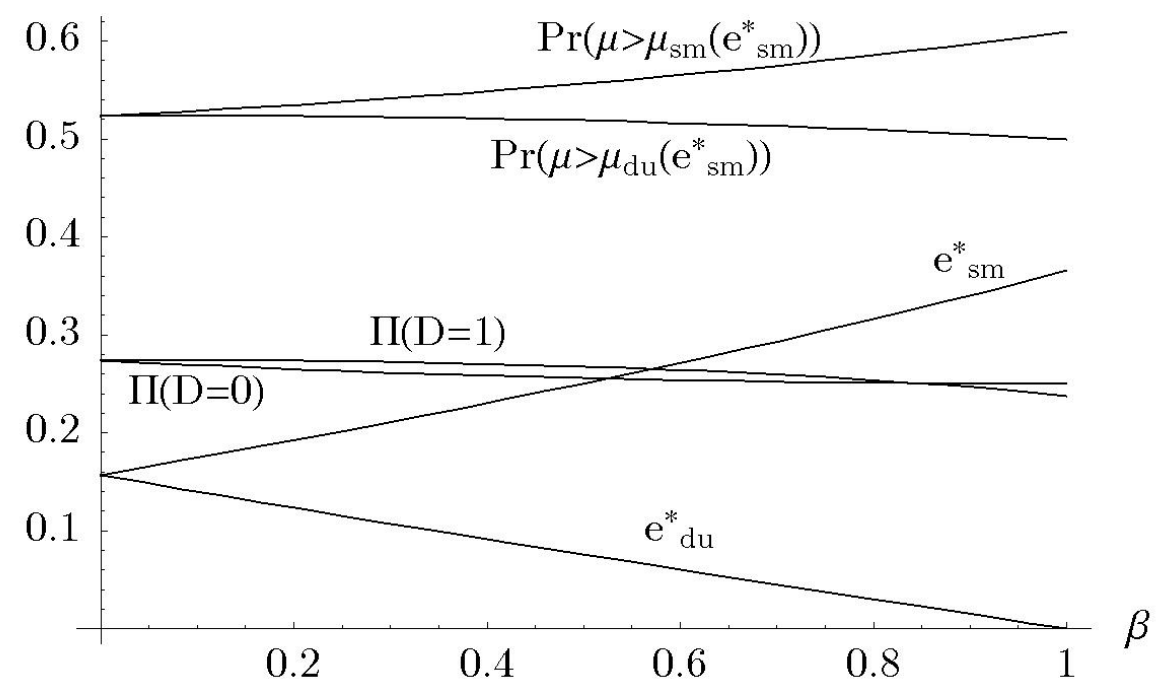

Figure 1: The motivation effect and the overconfidence effect and their net result on the incentives to delegate in case of $s=d u\left(c(e)=\frac{1}{2} e^{2}, \pi(e)=e, p=0, h=1, f=0.6\right.$ and $\eta=0.5)$.

Figure 1 illustrates how the motivation effect and the overconfidence effect together determine whether delegation can be used as a communication device. It is drawn under the assumption that the cost function is given by $c(e)=\frac{1}{2} e^{2}$ and that $\pi(e)=e$. Furthermore, we have assumed that $p=0, h=1, f=0.6$ and $\eta=0.5{ }^{10}$ Three pairs

\footnotetext{
${ }^{10}$ One can readily check that for these parameter values the senior would ex ante prefer a separating equilibrium to a pooling equilibrium.
} 
of lines are shown. First, the figure shows the optimal effort levels $e_{s m}^{*}$ and $e_{d u}^{*}$ exerted by a junior who believes he is smart with probability $\gamma_{s m}$ and $\gamma_{d u}$, respectively. For completely uninformative signals, $\beta=0$, these effort levels are identical. The difference in effort grows with any improvement in signal accuracy, and it becomes maximal for $\beta=1$. If a junior believes he is smart with probability $\gamma_{d u}=0$, he does not exert any effort at all. It is this difference in effort levels the senior is tempted to exploit by delegating the decision to implement the project to the junior if in fact her signal is $s=d u$. This gap in effort levels therefore captures the motivation effect. The overconfidence effect can be seen from the difference in probability of acceptance of the project, $\operatorname{Pr}\left(\mu>\mu_{s m}\left(e_{s m}^{*}\right)\right)>\operatorname{Pr}\left(\mu>\mu_{d u}\left(e_{s m}^{*}\right)\right)$. If the senior has received signal $s=d u$ and if the junior has exerted $e_{s m}^{*}$ effort, the correct probability of acceptance is $\operatorname{Pr}\left(\mu>\mu_{d u}\left(e_{s m}^{*}\right)\right)$. However, by delegating the implementation decision to the junior, the latter thinks he is smart with probability $\gamma_{s m}$, and this in turn gives rise to too large a probability of acceptance. The net result of the motivation and overconfidence effect is captured by the third pair of lines. Both are drawn conditional on $s=d u$. The line labelled $\Pi(D=1)$ shows the senior's payoffs in case she delegates the decision to implement to the junior, whereas the one labelled $\Pi(D=0)$ depicts payoffs if the senior makes the implement decision herself. For low values of $\beta>0$ the senior cannot withstand the temptation to delegate as she benefits more from the motivation effect than the overconfidence effect hurts her. For large values of $\beta$, the overconfidence effect is known to be too strong, and therefore $\Pi(D=0)>\Pi(D=1)$.

The upshot is that the presence of latent costs that would only become manifest in case the senior were to delegate the implementation decision when $s=d u$ make communication about the junior's ability possible. Recall that the base communication game is a cheap talk game in which no separating equilibrium exists due to the fact that the junior's costs of effort do not enter the senior's utility function. In that 
situation, if the junior believes the senior's announcement, no costs can withhold the senior from claiming that $s=s m$. However, delegating the implementation decision to a junior when $s=d u$ would hurt the senior. If these costs are large enough, the senior is no longer tempted to exaggerate the junior's ability. Interestingly, the latent nature of these costs is sufficient to withhold the senior from exaggerating. Delegation is therefore a costless, non-verbal signal. Accordingly, if Eq (6) holds, the first best solution is attained. That is, for given value of $s$, both the effort levels and the project implementation decisions are optimal.

Proposition 2 If a separating equilibrium exists (i.e., if $E q(6)$ holds), the senior's information is costlessly signalled, and the effort levels are first best.

Apart from the signalling reason, it does not matter who makes the implementation decision in our model. In many situations, however, it is likely that less experienced persons make more mistakes. In the context of our model this would mean that for given level of effort, delegation of the implementation decision to a junior increases the probability of a wrong implementation decision. The introduction of this aspect into our model has two effects. First, it makes delegation a less attractive device for improving communication between the senior and the junior. The benefit of a separating equilibrium must exceed the cost of more mistakes. Second, conditional on the senior preferring a separating equilibrium to a pooling one, the fact that a junior is an inferior decision maker widens the range of parameters for which a separating equilibrium exists. The reason is that it weakens the senior's incentive to delegate the implementation decision to a junior in case of $s=d u{ }^{11}$ This is important as it is this incentive that makes communication between the senior and the junior hard.

Let us conclude this section with a remark. Organizations tend to have a document,

\footnotetext{
${ }^{11}$ The expression at the right hand side of the inequality in Eq (6) decreases, making it more likely that the condition holds.
} 
like a manual of authority, that details how authority is delegated to positions within the organization. It does so by specifying what someone because of his or her position or office in the organization is allowed to decide on, how much money may maximally be involved depending on whether the outlays should be expensed or capitalised etc. From a communication perspective, our findings point to the fact that rulebound delegation complicates credible communication as it leaves little room for managerial discretion whether to delegate a task or not. In this respect, our paper is related to Simon (1951), who pointed out that a typical employment contract does not specify exactly what the employee should do. The rationale we provide differs from the one given by Simon. He focused on the advantage of postponing decision making such that the uncertain future can unfold.

\section{Collaboration}

In the introduction we mentioned various ways in which managers may dedicate time and attention to the design of the project or to the subordinate himself. One could imagine that part of this time directly improves the likelihood of success through 'collaboration', whereas the remaining part consists of, say, 'attention' that is not directly productive but that may nevertheless be used to signal valuable information about the senior's view concerning the quality of the junior. Attention can take on the form of, for instance, the provision of verbal (re-)assurance, invitations to meetings, and sharing of organizational gossip.

This section and the next deal with the question whether the senior can convey information about the junior's ability by collaborating with the junior or dedicating attention to him. We extend our base communication game by giving the senior the

opportunity to dedicate either form of time. Let $e^{B}$ stand for the total amount of time dedicated to the project by the senior, of which $\alpha e^{B}$ refers to collaboration and 
$(1-\alpha) e^{B}$ denotes attention. We maintain the assumption that when the junior is dumb, $a=D U$, the project is always bad, regardless of the effort the junior and/or the senior put in designing a project. A necessary condition for a good project therefore remains that the junior is smart, $a=S M$. Thus, if $a=S M$, then by collaborating with the junior, the senior increases the probability that the junior designs a good project. We assume that the senior expends effort before the junior, or that she can commit herself to an effort level. The probability that a good project is designed if a junior is smart, $a=S M$, equals $\pi\left(e^{J}, \alpha e^{B}\right)$ where $e^{J}$ stands for the effort exerted by the junior. ${ }^{12}$ We assume that $\pi_{J}>0, \pi_{B}>0$, and $\pi_{J B} \geq 0$, where subscripts refer to partial derivatives. Costs of effort are convex for both senior, $C\left(e^{B}\right)$, and junior, $c\left(e^{J}\right)$. To allow for the possibility that the senior's collaborative effort comes over and above his other duties, we assume $C(0) \geq 0$ and $C^{\prime}(0) \geq 0$. The payoff to the senior equals the revenue of the project, if implemented, minus the cost of her effort. In this section we analyse the case where all effort exerted by the senior is collaborative, $\alpha=1$. In Section 6, we turn to the situation in which she limits herself to paying attention, $\alpha=0$.

Our basic game had no separating equilibrium as it involved a cheap talk game in which players' interests differed too much. As a result, the junior did not learn the signal received by the senior. Does the extended game have a separating equilibrium? A necessary condition for a separating equilibrium of the extended game is that the senior's effort level depends on her type: the messages the senior sends can now be identified with the effort levels chosen. Let $e_{s m}^{B}$ and $e_{d u}^{B}$ denote the senior's equilibrium effort levels when she received signal $s=s m$ and $s=d u$, respectively. Roughly speaking, ${ }^{13}$ as the senior's effort is to no avail when $a=D U$, in a separating equilibrium we must have that $e_{s m}^{B}>e_{d u}^{B}$. Beliefs the junior holds regarding the information the

\footnotetext{
${ }^{12}$ We assume that the parameters are such that in any equilibrium $0<\pi\left(e^{J}, \alpha e^{B}\right)<1$.

${ }^{13}$ We make this statement precise in Lemma 4.
} 
senior has concerning his ability are ' $s=s m$ ' if $e_{B} \geq e_{s m}^{B}$ and ' $s=d u$ ' if $e_{B}<e_{s m}^{B}$. The last condition for a separating equilibrium is that a senior with signal $s=d u$ does not have an incentive to mimic the behaviour of a senior with signal $s=s m$.

We proceed as follows. In Subsection 5.1, we characterize the junior's effort level and the effort levels of both types of the senior assuming that when the junior makes his effort decision he knows the senior's type. Then we check whether the senior's effort levels satisfy the separating condition. Finally we turn to the question what the senior could do when the effort levels do no prevent him from exaggerating a junior's ability. As this subsection is based on general functional forms, we only derive implicit statements for equilibrium effort levels. To gain further insight, we turn to a special case in Subsection 5.2.

\subsection{Characterizing Equilibria}

We start by assuming that the junior knows the senior's type, i.e., by assuming the junior believes the senior's message. When the senior makes his decision about the project, implementation yields an expected payoff equal to $p+\gamma_{s} \pi\left(e_{s}^{J}, e_{s}^{B}\right) f+\mu$, where $e_{s}^{J}$ denotes the junior's effort conditional on the senior's type $s$. We write $e_{s}^{B}$ to highlight that the effort level $e^{B}$ signals type $s$. Implementation yields a higher payoff than maintaining the status quo if $\mu>\mu_{s}\left(e_{s}^{J}, e_{s}^{B}\right)=-p-\gamma_{s} \pi\left(e_{s}^{J}, e_{s}^{B}\right) f$.

The junior knows the senior's effort when determining his effort and anticipates the senior's decision rule. Expected project revenue when the junior decides how much effort to exert equals

$$
\begin{aligned}
R\left(e_{s}^{J}, e_{s}^{B} ; s\right) & =\operatorname{Pr}\left(\mu>\mu_{s}\left(e_{s}^{J}, e_{s}^{B}\right)\right) E\left(p+\gamma_{s} \pi\left(e_{s}^{J}, e_{s}^{B}\right) f+\mu \mid \mu>\mu_{s}\left(e_{s}^{J}, e_{s}^{B}\right)\right) \\
& =\operatorname{Pr}\left(\mu>\mu_{s}\left(e_{s}^{J}, e_{s}^{B}\right)\right)\left(-\mu_{s}\left(e_{s}^{J}, e_{s}^{B}\right)+\frac{1}{2}\left(h+\mu_{s}\left(e_{s}^{J}, e_{s}^{B}\right)\right)\right) \\
& =\frac{\left(p+h+\gamma_{s} \pi\left(e_{s}^{J}, e_{s}^{B}\right) f\right)^{2}}{4 h}
\end{aligned}
$$


The junior's best response, conditional on the senior's type $s, e_{s}^{J}\left(e_{s}^{B}\right)$, results from maximizing $R\left(e_{s}^{J}, e_{s}^{B} ; s\right)-c\left(e_{s}^{J}\right)$. An interior solution satisfies ${ }^{14}$

$$
R_{J}\left(e_{s}^{J}, e_{s}^{B} ; s\right)-c^{\prime}\left(e_{s}^{J}\right)=0
$$

Two features of the best response $e_{s}^{J}\left(e_{s}^{B}\right)$ that is implicitly defined by Eq (12) are worth noting. First, in case $e_{s}^{J}\left(e_{s}^{B}\right)>0$, higher levels of the senior's effort lead to higher levels of the junior's effort. Eq (10) and the expression for $\mu_{s}$ show why. The probability of acceptance as well as the expected payoff conditional on acceptance rise in effort, whether exerted by the junior or by the senior. Because we assumed that $\pi_{J B} \geq 0$, it follows that if the senior's effort increases, the marginal revenue of the junior's effort rises. Second, for $e_{s}^{J}\left(e_{s}^{B}\right)>0$ with $s \in\{s m, d u\}$, the larger is the likelihood $\gamma_{s}$ that the junior is smart, the larger is the marginal productivity of effort, and therefore the more effort the junior exerts for a given level of the senior's effort. In particular, $e_{s m}^{J}\left(e_{s}^{B}\right)>e_{d u}^{J}\left(e_{s}^{B}\right)$ for all $e_{s}^{B}$.

Lemma 3 Suppose that the junior knows the senior's type, and suppose $e_{s}^{J}\left(e_{s}^{B}\right)>0$ for $s \in\{s m, d u\}$. Then (i) the junior's effort is increasing in the senior's effort; and (ii) the junior's effort is increasing in the probability that he is smart, implying $e_{s m}^{J}\left(e_{s}^{B}\right)>e_{d u}^{J}\left(e_{s}^{B}\right)$ for all $e_{s}^{B}$.

Now consider the effort decision the senior has to make. The senior takes into account the effect her choice has on the junior's effort level. That is, the senior maximizes

$$
R\left(e_{s}^{J}\left(e_{s}^{B}\right), e_{s}^{B} ; s\right)-C\left(e_{s}^{B}\right), \text { for } s \in\{s m, d u\}
$$

and so the first order condition for an interior solution becomes

$$
R_{J}\left(e_{s}^{J}\left(e_{s}^{B}\right), e_{s}^{B} ; s\right) \frac{d e_{s}^{J}}{d e_{s}^{B}}+R_{B}\left(e_{s}^{J}\left(e_{s}^{B}\right), e_{s}^{B} ; s\right)=C^{\prime}\left(e_{s}^{B}\right)
$$

\footnotetext{
${ }^{14}$ We assume that the second order condition $R_{J J}\left(e_{s}^{J}, e_{s}^{B} ; s\right)-c^{\prime \prime}\left(e_{s}^{J}\right)<0$ holds.
} 
Because the senior undertakes a host of activities, it may well be that collaborating with the junior is too expensive or not rewarding enough. In that case the senior does not exert any effort if she is of type $s$ as

$$
R_{J}\left(e_{s}^{J}(0), 0 ; s\right) \frac{d e_{s}^{J}}{d e_{s}^{B}}+R_{B}\left(e_{s}^{J}(0), 0 ; s\right) \leq C^{\prime}(0)
$$

Let $\left(e_{s m}^{* B}, e_{d u}^{* B}\right)$ denote the pair of effort levels such that $e_{s}^{* B}$ satisfies either Eq (14) or (15) for $s$. If $\left(e_{s m}^{* B}, e_{d u}^{* B}\right)>0$, the question becomes whether the senior exerts more effort in case of $s=s m$ or in case of $s=d u$. Clearly, as the marginal revenue of effort of both the junior and the senior increase in the perceived ability of the junior, the answer to this question hinges on the way $\gamma_{s}$ influences $d e_{s}^{J} / d e_{s}^{B}$. To guarantee that a senior who is more confident to collaborate with a smart junior exerts more effort than in case she were less confident, we impose a mild restriction on the way $d e_{s}^{J} / d e_{s}^{B}$ changes with an increase in $\gamma$. Lemma 4 states that as long as $\pi_{J J}$ is not 'too negative', $e_{s m}^{* B}>e_{d u}^{* B}$. Hence, the more able the senior believes the junior to be, the more effort she exerts.

Lemma 4 Suppose an interior solution $e_{s m}^{* B}$ exists for $s=s m$. Suppose $\pi_{J J}$ is not too negative. Then, $e_{s m}^{* B}>e_{d u}^{* B}$.

To highlight the fact that $\left(e_{s m}^{* B}, e_{d u}^{* B}\right)$ has been derived ignoring any separating condition and looking only at the productive side of the senior's effort, we refer to these levels as 'productive effort levels'. These effort levels $\left(e_{s m}^{* B}, e_{d u}^{* B}\right)$ reveal the senior's type if they are such that the senior has no incentive to choose $e_{s m}^{* B}$ when her type is $s=d u$. In other words, the pair $\left(e_{s m}^{* B}, e_{d u}^{* B}\right)$ is part of a separating equilibrium if it satisfies the separating condition

$$
R\left(e_{d u}^{J}\left(e_{d u}^{B}\right), e_{d u}^{B} ; d u\right)-C\left(e_{d u}^{B}\right) \geq R\left(e_{s m}^{J}\left(e_{s m}^{B}\right), e_{s m}^{B} ; d u\right)-C\left(e_{s m}^{B}\right)
$$

where $R\left(e_{s m}^{J}\left(e_{s m}^{B}\right), e_{s m}^{B} ; d u\right)$ stands for the revenue the senior obtains when $s=d u$ and bases her implementation decision on $\gamma_{d u}$ rather than $\gamma_{s m}$, but signals $s=s m$ 
by exerting $e_{s m}^{B}$ effort. Obviously, if $e_{s m}^{* B}=e_{d u}^{* B}=0$, the separating condition is not satisfied. Define $e_{s}^{* J}:=e_{s}^{J}\left(e_{s}^{* B}\right)$ for $s \in\{s m, d u\}$.

Proposition 3 Let $e_{s}^{* B}$ solve $E q(14)$ or (15) for $s \in\{d u, s m\}$. If the pair $\left(e_{s m}^{* B}, e_{d u}^{* B}\right)$ satisfies the separating condition (16) then it is part of a separating equilibrium. In this equilibrium, the junior believes that $s=s m$ if and only if $e^{B} \geq e_{s m}^{* B}$, and exerts effort $\left(e_{s m}^{* J}, e_{d u}^{* J}\right)$. In any other case (i.e., $e_{s m}^{* B}>0$ but condition (16) is violated, or $\left.e_{s m}^{* B}=0\right)$, $e_{s m}^{* B}$ and $e_{d u}^{* B}$ are not part of a separating equilibrium.

Proposition 3 states that if condition (16) holds the junior learns the senior's type. Important for this result is that time spent on a junior in case of a favourable assessment is more productive than in case of a less favourable assessment. A direct implication is that it is costly for the senior to make exaggerated claims as to the junior's ability. The separation condition is satisfied, if the costs of mimicking exceed the benefits.

What if the productive effort levels $\left(e_{d u}^{* B}, e_{s m}^{* B}\right)$ do not satisfy the separation condition? As in Section 3, the senior's problem is that she then would like to make any junior believe that her assessment is $s=s m$. As a result, a junior observing $e_{s m}^{* B}$ does not believe that the senior really assesses her favourably. As usual in signalling models, the senior can make her message about her type credible by sending a costly message. In our model, this amounts to exerting that level of effort that ensures that mimicking if $s=d u$ is not beneficial. Hence, if $s=s m$, the senior exerts effort, $\hat{e}_{s m}^{B}$ so that Condition (16) holds with equality (with $e_{d u}^{* B}$ resulting from either Eq (14) or (15)). Given $\widehat{e}_{s m}^{B}$ and $e_{d u}^{* B}$, the junior believes that the senior's signal is $s=s m$ if and only if $e^{B} \geq \widehat{e}_{s m}^{B}$. The last condition for a separating equilibrium is that $\hat{e}_{s m}^{B}$ (with separating) yields a higher payoff than $e_{s m}^{* B}$ (inducing the junior to believe that the senior's type is $s=d u$, while it is in fact $s=s m$ ). 


\subsection{Analyzing Equilibrium Behaviour}

In this subsection we analyse a special case to shed light on the way key parameters influence the possibility of communication. We restrict our model in two ways. First, we consider a linear function $\pi\left(e^{J}, e^{B}\right)=e^{J}+e^{B}$. Second, we assume that the costs of effort are quadratic: for the junior, $c\left(e^{J}\right)=\frac{1}{2} \lambda e^{J 2}$, with $\lambda>0$, and for the senior $C\left(e^{B}\right)=\frac{1}{2} \lambda_{B}\left(e^{B}+\Omega\right)^{2}$, with $\lambda_{B}>0$ and $\Omega>0$.

Assume that the junior believes the senior's signal. The junior's best response function becomes (see Eq (12)):

$$
\begin{aligned}
e_{s}^{J}\left(e_{s}^{B}\right) & =\frac{(p+h) \gamma_{s} f}{2 h \lambda-\gamma_{s}^{2} f^{2}}+\frac{\gamma_{s} f}{2 h \lambda-\gamma_{s}^{2} f^{2}} e_{s}^{B} \\
& =A_{s}+B_{s} e_{s}^{B}, s \in\{s m, d u\}
\end{aligned}
$$

with $A_{s}=(p+h) \gamma_{s} f /\left(2 h \lambda-\gamma_{s}^{2} f^{2}\right), B_{s}=\gamma_{s} f /\left(2 h \lambda-\gamma_{s}^{2} f^{2}\right)$, and where $2 h \lambda-\gamma_{s}^{2} f^{2}>$ 0 because of the second order condition for a maximum. In particular, even if the senior were not to exert any effort, the junior would. Observe that the junior's effort is increasing in the effort exerted by the senior. Also note that, for a given level of effort of the senior, the more the junior believes being smart, the higher is his effort. Both statements follow from Lemma 3.

The senior takes this best response function into account when maximizing his payoff net of cost. Her objective function becomes

$$
\frac{1}{4 h}\left(p+h+\gamma_{s} f A_{s}+\gamma_{s} f\left(1+B_{s}\right) e_{s}^{B}\right)^{2}-\frac{1}{2} \lambda_{B}\left(e_{s}^{B}+\Omega\right)^{2}
$$

The senior maximizes expression (18), with respect to $e_{s}^{B}$, subject to $e_{s}^{B} \geq 0$. This yields the productive effort levels

$$
e_{s}^{* B}= \begin{cases}\frac{\Delta_{s}}{2 h \lambda_{B}-\gamma_{s}^{2} f^{2}\left(1+B_{s}\right)^{2}} & \text { if } \Delta_{s}>0 \\ 0 & \text { if } \Delta_{s} \leq 0\end{cases}
$$


where $\Delta_{s}=\left(1+B_{s}\right) \gamma_{s}\left(p+h+\gamma_{s} f A_{s}\right) f-2 h \lambda_{B} \Omega .{ }^{15}$ It is immediately clear that if $e_{s m}^{* B}>0$, then $e_{s m}^{* B}>e_{d u}^{* B}$, as implied by lemma 4 .

We now analyse how the accuracy $\beta$ of the senior's assessment of the junior's ability influences optimal effort levels. The senior does not exert effort if she knows that her effort is very unlikely to pay off. If the senior's signal accurately reveals the junior's ability, $\beta=1$, and the signal equals $s=d u$, then $\gamma_{d u}=0$. The marginal benefits of effort are therefore nil, whereas the marginal costs of effort equal at least $\lambda_{B} \Omega$. Under such circumstances, the senior does not exert effort. Define $\bar{\beta}$ as the value of $\beta \in[0,1)$ such that $\Delta_{d u}=0$. Then for sufficiently trustworthy information, $\beta \geq \bar{\beta}$, the optimal level of effort in case of $s=d u$ equals $e_{d u}^{B}=0$. As we illustrate below, $\bar{\beta}$ is not necessarily 'close' to one.

Lemma 5 Suppose the junior believes that the senior tells the truth, $s=m$. For $\beta \geq \bar{\beta}$, with $\bar{\beta}<1$, the senior does not exert effort if $s=d u, e_{d u}^{B}=0$.

Of course, effort should be sufficiently beneficial to be exerted even in case of a good signal, $s=s m$. The lower is the possible upside $f$ in case of a good project, the smaller is the set of parameters for which the senior exerts effort, both in case $s=d u$ and $s=s m$. Similarly, the higher are the cost of exerting effort, $\lambda_{B}$ and/or $\Omega$, the smaller is the set of parameters for which effort is exerted.

Our game revolves around the senior's inclination to exaggerate the junior's quality. An important factor that determines this inclination is the degree of signal accuracy $\beta$ through its effect on $\gamma_{s m}$ and $\gamma_{d u}$. Assume that for $\beta=0, e_{s m}^{* B}>0$. It follows from Eq (19) that $e_{s m}^{* B}$ increases in $\beta$. The reason is that the higher is the probability that the junior is smart, the higher is the expected marginal revenue of the senior's effort. The effect of $\beta$ on $e_{d u}^{* B}$, by contrast, is negative until $\beta=\bar{\beta}$. We have seen that for

\footnotetext{
${ }^{15}$ The denominator in the expression for $e_{s}^{B}$ if $\Delta_{s}>0,2 h \lambda_{B}-\gamma_{m}^{2} f^{2}\left(1+B_{m}\right)^{2}$, is positive by the second order condition for a maximum.
} 
values of $\beta \geq \bar{\beta}$ it equals zero. The spread of $e_{s m}^{* B}$ and $e_{d u}^{* B}$ thus increases in $\beta$. A direct implication is that also the costs of mimicking increases in $\beta$.

The benefit of exaggerating the ability of a junior is the concomitant increase in the junior's level of effort because of the change in belief he holds about his quality. The higher is $\beta$ the larger is this increase. However, any increase in $\beta$ implies a reduction in the likelihood that this additional effort will in fact be productive. When $\beta=1$, there are no benefits of mimicking.

More generally, the above discussion implies that there is a value $\widehat{\beta} \in[0,1)$ such that for $\beta \geq \widehat{\beta}$ the senior truthfully reveals what she knows about the junior as the benefits of mimicking fall short of the cost of the extra effort the senior would have to exert. If, however, $\beta \in[0, \widehat{\beta}]$, the only way to avoid exaggerating the junior's ability is by making exaggeration too costly, that is by exerting more effort if $s=s m$. This is illustrated in figure 2, which has been drawn for $p=0, h=1, f=0.5, \eta=0.5$, $\lambda=\lambda_{B}=1$, and $\Omega=0.1 .^{16}$

The figure highlights that the senior's effort $e_{d u}^{* B}$ decreases in $\beta$ and equals zero for $1 \geq \beta>\bar{\beta} \approx 0.28$. As a result, the senior's effort $e_{d u}^{* J}$ decreases in $\beta$ and equals zero for $\beta=1 .^{17}$ For $\beta<\widehat{\beta} \approx 0.36$, the senior's effort level $e_{s m}^{* B}$ that is optimal from a productive point of view differs too little from $e_{d u}^{* B}$ for the senior not to be tempted to exaggerate the junior's ability. The dotted section of the line labelled $e_{s m}^{* B}$ indicates that credible communication is not possible at these effort levels. The bulge-shaped solid line shows the extent to which the senior has to exert more effort in case her signal equals $s=s m$. As a result, the junior also exerts more effort than would be optimal from a productive point of view.

We now turn to a different element in our model, the factor $\Omega$ that appears in the

\footnotetext{
${ }^{16}$ One can readily check that for these parameter values the senior ex ante prefers a separating equilibrium to a pooling equilibrium.

${ }^{17}$ Moreover, there is a hardly noticeable kink in the graph at $\beta=\bar{\beta}$.
} 


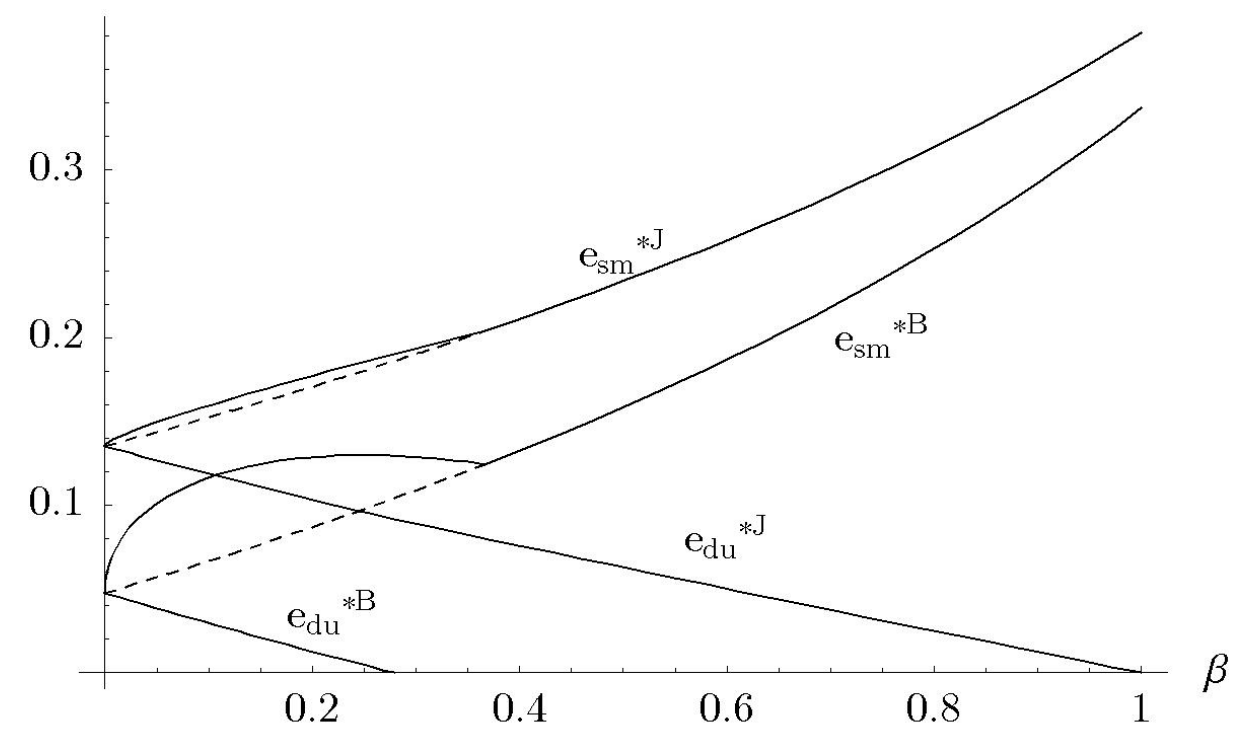

Figure 2: Optimal effort levels of senior and junior. Drawn lines satisfy separating condition, dotted line sections do not $\left(p=0, h=1, f=0.5, \eta=0.5, \lambda=\lambda_{B}=1\right.$, and $\Omega=0.1)$

senior's cost function. This factor could be interpreted as the extent to which the senior is burdened with other tasks. The larger is $\Omega$, the more time and attention the senior dedicates to other tasks. An increase in $\Omega$ has two effects on the productive effort levels given by Eq (19). The larger is $\Omega$, the larger are the 'start up' costs of exerting any effort at all. As a consequence, if for a given value of $\Omega$ and for a given level of signal accuracy, the senior does not exert effort in case of $s$, she does not exert effort either for higher values of $\Omega$. Secondly, the larger is $\Omega$, the larger are the marginal costs of exerting effort. This implies that the increase in effort stemming from increasing levels of signal accuracy goes down as $\Omega$ goes up. Because of either reason, the optimal effort levels of the junior decrease with any increase in $\Omega .{ }^{18}$ An important consequence of all this is that credible communication becomes more problematic with any increase in $\Omega$,

\footnotetext{
${ }^{18}$ For very high values of $\Omega$, the senior may prefer a pooling equilibrium to a separating one.
} 
as the levels of effort $e_{s m}^{* B}$ and $e_{d u}^{* B}$ become more and more similar (see Eq (19)). That is, the more overburdened the senior is by other tasks, the more often the senior has to deviate from the productive effort levels $\left(e_{d u}^{* B}, e_{s m}^{* B}\right)$ so as to convince the junior. This suggests that an organization that assigns multiple tasks to managers should take into account not only the costs and benefits of these tasks, but also the effect the workload of a manager has on her possibilities of revealing valuable information.

\section{Attention}

In this section we turn to the case where all time spent by the senior amounts to attention, rather than collaboration, $\alpha=0$. Recall that such effort is costly, but does not directly affect the chances of success of the project. If such effort is of any use in the present model, it is because of its signalling value.

We continue with the specific case of a linear $\pi$-function and quadratic costs. As the senior's temptation is to exaggerate the ability of a junior, the aim is to pay attention to a junior if one believes he is smart with probability $\gamma_{s m}$ and to ignore the junior in case of $\gamma_{d u}$. Let $e_{s m}^{* B}$ be the smallest level of effort, such that the separating condition

$$
\frac{1}{4 h}\left(p+h+\gamma_{d u} f A_{d u}\right)^{2}-\frac{1}{2} \lambda_{B} \Omega^{2}>\frac{1}{4 h}\left(p+h+\gamma_{d u} f A_{s m}\right)^{2}-\frac{1}{2} \lambda_{B}\left(e_{s m}^{B}+\Omega\right)^{2}
$$

is satisfied. Recall that $A_{s}=\frac{(p+h) \gamma_{s} f}{2 h \lambda-\gamma_{s}^{2} f^{2}}$ was obtained in Eq (17). Because the senior is tempted to exaggerate the junior's ability for $\beta \in(0,1)$, the senior has to pay attention in case of $s=s m$ to make her statement credible.

Figure 3 presents the relationship between $\beta$ and $e_{s m}^{* B}$, with $p=0, h=1, f=0.6$ and $\eta=\frac{1}{2}$. For low levels of $\beta$, the benefits of exaggerating the junior's ability are small. Little attention, $e_{s m}^{* B}$, is therefore needed to withhold the senior from exaggerating. With increasing levels of $\beta$, the increase in effort exerted by the junior thanks to his enhanced self confidence goes up. However, increasing values of $\beta$ reduce the likelihood 


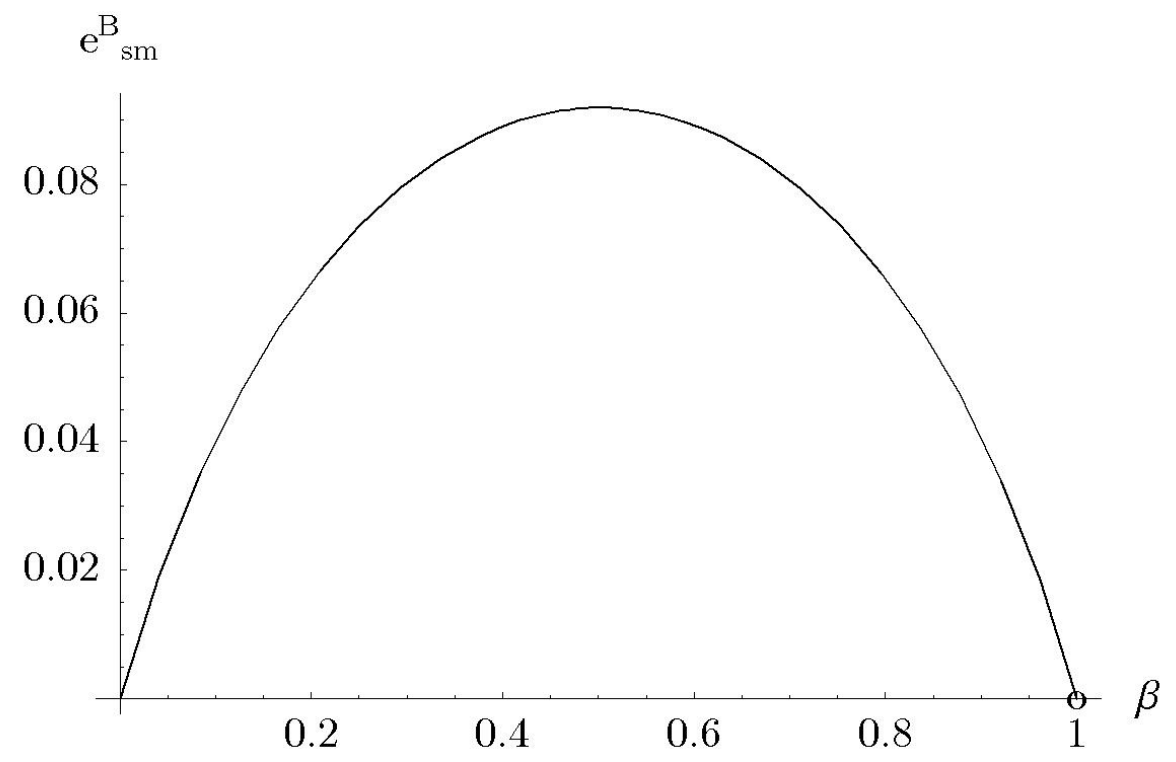

Figure 3: The amount of attention given by the senior in case $s=s m(p=0, h=1$, $f=0.6$ and $\eta=0.5)$.

that this additional effort will be beneficial. As a result, with $\beta$ going up from zero to one, attention starts out being rare, grows more intense, and then declines again to virtually disappear in case of flawless information-the most minimalist form of attention that is given exclusively in case of $s=s m$ suffices to make communication credible.

\section{Concluding Remarks}

Sometimes seniors have better information about juniors' abilities than the juniors themselves. In those situations it is not always obvious for a senior to share her information by ordinary talk. We have explained why. When ability and effort are complements, the senior has an incentive to exaggerate a junior's ability. The implication is that the senior's words do not depend on her perception of the junior's ability. As a consequence, her words do not contain information. 
From the signalling literature we know that if talk does not work, then revealing information may be possible through costly signals. This was the topic of the last part of our article. We have shown that a smart type senior-i.e., one who has a favourable impression of the junior-can engage in costly collaboration or attention beyond what a dumb type of senior (one who has a less favourable impression) would want to mimic. It is worth mentioning that this result could have been obtained in a model in which only one task, involving costly effort, has to be performed. Indeed, using such a model, Hermalin (1998) has shown that by working harder than what would be optimal from a purely productive point of view, a senior can signal her private information about a productivity factor. ${ }^{19}$

The existence of a second task is essential for our result that the senior can prevent herself from exaggerating the junior's ability by delegating the implementation decision only to juniors she deems able. Why does not a senior with a less favourable impression also delegate the implementation decision to the junior? The reason is that the senior wants the person who makes the implementation decision to have a correct perception of the quality of the project. In a separating equilibrium of the model with delegation, exaggerating a junior's ability leads to more effort, but also to a distorted implementation decision. Thus, apart from benefits there are now costs of exaggerating a junior's ability. If these costs exceed the benefits, the senior can reveal her information to the junior.

We have assumed that if the junior possesses the same information as the senior, he is equally able to take the implementation decision. Thus, in a separating equilibrium in which delegation only takes place if the senior's impression is favourable, the junior takes the same decision as the senior. ${ }^{20}$ As discussed, our result does not hinge on this

\footnotetext{
${ }^{19}$ Benabou and Tirole (2002) also focus on one task.

${ }^{20} \mathrm{In}$ a pooling equilibrium, the senior is a better decision maker. As the senior has superior information about the junior's ability, she can better assess the quality of the project.
} 
assumption. Our reason for making this assumption was to highlight that there are not necessarily costs involved in revealing information through delegation. ${ }^{21}$ Because we analyse collaboration in a model with two tasks, we can compare its desirability with that of delegation as a means of communication. As signalling through collaboration is costly, whereas communication through delegation is not, an implication in our model is that delegation, if feasible, is superior to communication through attention.

\section{References}

Aghion, Phillipe and Jean Tirole (1997), Formal and Real Authority in Organizations, Journal of Political Economy, 105, 1-29.

Bénabou, Roland and Jean Tirole (forthcoming), Intrinsic and Extrinsic Motivation, Review of Economic Studies.

Calvo, Guillermo and Stanislaw Wellisz (1978), Supervision, Loss of Control, and the Optimum Size of the Firm, Journal of Political Economy, 86, 943-952.

Farrell, Joseph and Matthew Rabin (1996), Cheap Talk, Journal of Economic Perspectives, $10(3)$, 103-118.

Garicano, Luis (2000), Hierarchies and the Organization of Knowledge in Production, Journal of Political Economy, 108, 874-904.

Hermalin, Benjamin (1998), Toward an Economic Theory of Leadership: Leading by Example, American Economic Review, 88 (5), 1188-1206.

\footnotetext{
${ }^{21}$ We have therefore shown that one could induce credible communication without incurring costs. This possibility is not common. Farell and Rabin (1996, p. 106), for example, discuss a cheap talk game in which the employee is informed about her ability but his future employer is not. As their payoffs are such that cheap talk cannot convey the employee's private information, Farell and Rabin conclude that "either no information will be conveyed, or [the employee] will engage in costly signalling".
} 
Marschak, Jacob and Roy Radner (1972), Economic Theory of Teams. New Haven: Yale University Press.

Mintzberg, Henry (1971), Managerial Work: Analysis from Observation, Management Science, 18 (2), B97-B110.

Prendergast, Canice (1999), The Provision of Incentives in Organizations, Journal of Economic Literature, 37, 7-63.

Simon, Herbert (1951), A Formal Theory of the Employment Relationship, Econometrica, 19 (3) 293-305.

Zábojnik, Ján (2002), Centralized and Decentralized Decision Making in Organizations, Journal of Labor Economics, 20, 1-22.

\section{Appendix}

Proof of Proposition 1: The beliefs of the junior are clearly consistent with the proposed equilibrium strategies of either type of senior. Given these beliefs, in case of $D=1$, the junior maximizes $\frac{1}{4 h}\left(p+h+\pi(e) \gamma_{s m} f\right)^{2}-c(e)$ and so $e_{s m}^{*}$ satisfies Eq (8) for $s=s m$, and the junior implements the project if and only if condition (7) holds. Similarly, in case of $D=0, e_{d u}^{*}$ satisfies Eq (8) for $s=d u$. Given this strategy of the junior, and given that condition (6) holds, the senior's best reply is $D=1$ in case of $s=s m$ and $D=0$ in case of $s=d u$. In the latter case, she decides to implement if and only if condition (7) is met for $s=d u$. Note that condition (6) says that if $s=d u$ the payoff of not delegating the implementation decision is larger than the payoff of delegating this decision.

Proof of Lemma 3: To show part (i), use the first order condition for a maximum in $e_{s}^{J}$ to obtain

$$
d e_{s}^{J}\left[R_{J J}\left(e_{s}^{J}, e_{s}^{B} ; s\right)-c^{\prime \prime}\left(e_{s}^{J}\right)\right]+d e_{s}^{B}\left[R_{J B}\left(e_{s}^{J}, e_{s}^{B} ; s\right)\right]=0
$$


The first expression in square brackets is negative by the second order condition. The second term in square brackets is positive by our assumption that $\pi_{J B} \geq 0$. Hence, $d e_{s}^{J} / d e_{s}^{B}>0$ on the best reply function. Part (ii) follows immediately as for any level of effort exerted by the senior, the marginal productivity increases if the junior believes the senior's level of effort signals $s=s m$ rather than $s=d u$. QED

Proof of Lemma 4: Suppose $e_{s m}^{* B}>0$. If $e_{d u}^{* B}=0$, we're done. So, assume $e_{d u}^{* B}>0$. Eq (14) now holds for both types of seniors. The partial derivatives $R_{B}$ and $R_{J}$ are increasing in $\gamma_{s}$. To prove the lemma, it is sufficient to show that $d e_{s}^{J} / d e_{s}^{B}$ also increases in $\gamma_{s}$. Formally,

$$
\frac{d e_{s}^{J}}{d e_{s}^{B}}=-\frac{R_{J B}}{R_{J J}-c^{\prime \prime}}
$$

Therefore

$$
\frac{\partial \frac{d e_{s}^{J}}{d e_{s}^{B}}}{\partial \gamma_{s}}=-\frac{\frac{\partial R_{J B}}{\partial \gamma_{s}}\left(R_{J J}-c^{\prime \prime}\right)-\frac{\partial R_{J J}}{\partial \gamma_{s}} R_{J B}}{\left(R_{J J}-c^{\prime \prime}\right)^{2}}
$$

Furthermore

$$
\begin{aligned}
\frac{\partial R_{J B}}{\partial \gamma_{s}} & =\frac{\gamma_{s} f}{h} \pi_{B} \pi_{J}+\frac{(p+h) f}{2 h} \pi_{J B}+\frac{\gamma_{s} \pi f^{2}}{h} \pi_{J B}>0 \\
\frac{\partial R_{J J}}{\partial \gamma_{s}} & =\frac{\gamma_{s} f^{2}}{h} \pi_{J} \pi_{J}+\frac{(p+h) f}{2 h} \pi_{J J}+\frac{\gamma_{s} \pi f^{2}}{h} \pi_{J J}
\end{aligned}
$$

As $R_{J J}-c^{\prime \prime}<0$ by the second order condition for a maximum, and $\partial R_{J B} / \partial \gamma_{s}>0$, the derivative in equation (A. 3) is positive if $\pi_{J J}$ is not too negative. QED 